\title{
Optimization of urban scenic spot planning based on ecological chain and reversed-planning theory-Using Yunlong Lake in Xuzhou city as an example
}

\author{
Lan Zhang ${ }^{1}$, Difei Zhao ${ }^{2, *}$, Rui $\mathrm{Li}^{3}$, and Yingxing $\mathrm{Chen}^{4}$ \\ ${ }^{1}$ School of Architecture and Design, China University of Mining and Technology, Xuzhou, Jiangsu 221116, China \\ 2 Artificial Intelligence Research Institute, China University of Mining and Technology, Xuzhou, Jiangsu 221116, China \\ ${ }^{3}$ School of Foreign Studies, China University of Mining and Technology, Xuzhou, Jiangsu 221116, China \\ ${ }^{4}$ School of History, Culture\&Tourism, Huaiyin Normal University, Huaian, Jiangsu 223300, China
}

\begin{abstract}
Modernized urban organization relies more on the foundation of ecological infrastructure (EI) with the planning objectives based more on synthetical benefits of economy, society and ecological environment. The effect of multiple factors ought to be taken into consideration in the urban scenic spot planning and and the process of formulating the related plan faces a challenge of how to organically bound environmental factors to tour function of urban scenic spot. This thesis, taking YunLong Lake, a scenic spot in XuZhou, JiangSu province as an example, studies how to ameliorate the planning of urban scenic spot combined with the ecological chain and reversed-planning theory. The result shows that utilizing the theory flexibly when analyzing the urban scenic spot planning design can ensure the existing ecological connection between each system and maintain the ecological stability of urban scenic spot. Thus, with the basis of ecological chain and reversed-planning theory, this paper puts forward a new planning concept and work flow, in order to provide new ideas for modernized urban scenic spot planning.
\end{abstract}

\section{Introduction}

Sustainable development is the coordinated development of economy, society and ecological environment [1]. Whereas, in the current urban planning and design, the emphasis on economic factors evidently higher than that on social and ecological one, which leads to a series of natural and social environment problems. Lake or wetland scenic spot, one part of modern cities, is a vital button to ameliorate the urban ecological environment [2-4]. How to unify the environmental benefits into tourism functions in urban scenic spots has become a puzzle faced by urban scenic spots planning. In recent years, the scientific idea of ecological environment has also been applied in the planning of urban scenic spots. Environmental design concepts such as imitating natural community design, artificially assisting natural succession, and regulating biological chains through biological control have also been utilized in the design and planning of urban scenic spots so as to optimize and improve the environmental functions of urban scenic spots. Accordingly, only when all function of urban scenic spots obtains the specific design in planning does each corresponding function can be ensured in operation after the completion of the design. This paper, taking YunLong Lake, a scenic spot in XuZhou, JiangSu province as an example, by synthetically considering the impact of natural environment on scenic spot planning, raises a new approach of connecting ecological chain and reversed-planning concept in modernized urban scenic spots.

\section{Research Objects and Theoretical Basis}

\subsection{Overview of Yunlong Lake Scenic Spot in Xuzhou}

Yunlong Lake Scenic Spot is located in the southern part of Xuzhou City (Fig. 1), covering an area of 44.7 square kilometers. It was approved as a national AAAAA-level scenic spot in August 2016. Yunlong Lake featuring the natural landscape, is a top urban scenic spot surrounded by mountains on three sides and a city on one side (Fig. 2), with Han culture, celebrity culture, and religious culture as the main content and also equipped with comprehensive functions such as sightseeing and leisure.

\subsection{Ecological chain}

Ecosystem refers to a unified whole in which all creatures inhabiting together in a certain area (i.e. biotic community) interact mutually and interdependently with their nonbiological environment through continuous material circulation and energy flow process [5-6]. In order to sustain the ecological balance in system by employing a

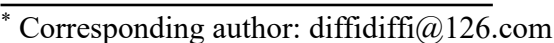


self-regulating mechanism, the biological components in the ecosystem are directly or indirectly connected through the food web, maintaining the relative stability of the structure and function of the ecosystem. Whereas, the selfregulation capacity of the ecosystem is limited and the adjustment wouldn't take effect well once beyond the limitation. Furthermore, the ecological system of urban lake scenic spots is greatly affected by the city planning, mainly showing the marked effect on the ecological chain of lake scenic spots caused by artificial modification.

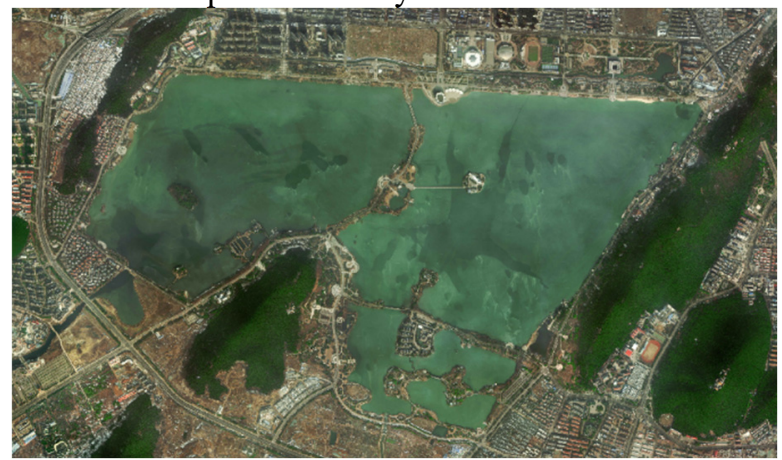

Fig. 1. Yunlong lake scenic spot in Xuzhou city

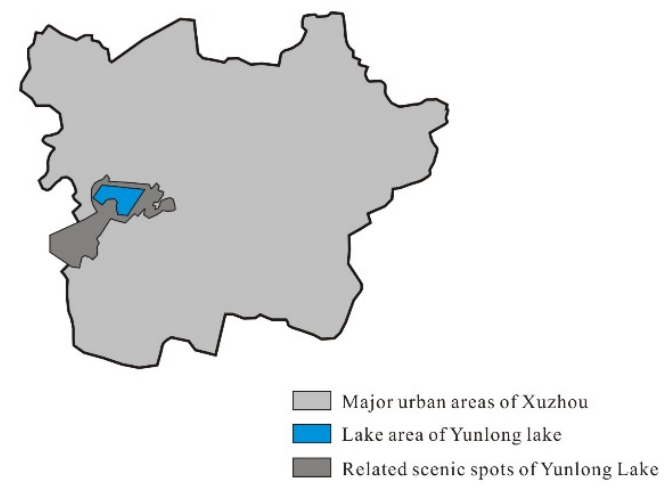

Fig. 2. The relationship between Yunlong lake, related scenic spots and Xuzhou city

\subsection{Reversed-planning theory}

Early planning, influenced by modernist architectural thoughts born in the industrial age which pursues function and efficiency, tend to consider economic benefits onesidedly and ignore other factors such as ecology and humanity [7]. It is "reversed-planning " theory that criticized and reflected on traditional planning lacking of ecological consciousness [1]. Prior to the traditional planning due to its comprehensive consideration of various ecological factors, " Reversed-planning " refers to the first planning formulated for non-constructible land and restricted exploitation land on the basis of protecting the original ecological pattern or enabling it to display greater ecological benefits and as a result, forming a mandatory guidance for usable area. Traditional planning regards usable area as figure and non-constructive land as background in most cases, thus neglecting the significance of non-constructive land and even making it a negative element. While "reversed-planning "can coordinate the relationship between ecological environment and construction in the condition of not destroying the original ecological pattern and even generating its greater benefits.

\section{New approach of Urban Scenic Spots Planning Based on Ecological Chain and Reversed-planning Theory}

There exists discrepancy in the ecological chain between urban scenic spots and the natural environment. The remarkable impact of human activities in city as well as artificial buildings in scenic spots on the natural ecological environment of urban scenic spots weaken the already fragile ecological chain. Yet as for modern cities, urban scenic spots are still a vital functional unit of ecological regulation, and also a crucial human environment regulation unit. Consequently, the urban lake area landscape is able to function well in tourism economy, ecological and humanistic environment, only within the special ecological environment marked with urban human behaviours, that is, maintaining the stability of ecological environment function can maximize its tourism economic and humanistic environment effect. To sum up, the result of this research suggests that when planning and designing urban scenic spots, a sustainable and stable ecological chain ought to be fully understood and created first with the basis of sustaining the stability of urban ecological environment. What's more, gaining the environmental and ecological benefits of scenic spots planning requires further synthetically taking the impact of human factors into account while designing the ecological chain [8]. In the perspective of the ecological benefits of scenic spots, the research proposes a new design workflow as follows by the combination of the ecological chain and reversedplanning concepts:

Table 1. Work flow of urban scenic spots planning based on ecological chain and reversed-planning theory

\begin{tabular}{|c|c|}
\hline Step & Function \\
\hline $\begin{array}{c}\text { Ecological chain } \\
\text { study }\end{array}$ & $\begin{array}{c}\text { To understand the ecosystem } \\
\text { and key influencing factors of } \\
\text { scenic spots }\end{array}$ \\
\hline $\begin{array}{c}\text { Planning of non- } \\
\text { constructive land } \\
\text { and restricted } \\
\text { construction land }\end{array}$ & $\begin{array}{c}\text { To ensure the urban lake area } \\
\text { can function well in } \\
\text { ecological level }\end{array}$ \\
\hline $\begin{array}{c}\text { Planning of } \\
\text { constructive land }\end{array}$ & $\begin{array}{c}\text { To ensure that the scheme } \\
\text { takes into account the } \\
\text { environmental function and } \\
\text { the scenic spot tour function }\end{array}$ \\
\hline $\begin{array}{c}\text { Synthetically } \\
\text { ameliorate the } \\
\text { planning and } \\
\text { design planning }\end{array}$
\end{tabular}

1) In the first place, we should investigate the urban scenic spots and surrounding environment by combining the regional characteristics of ecological environment so as to complete the design of ecological chain. What's more, the impact of man-made regulation factors on the urban lake landscape in the design of ecological chain demands to be taken into account;

2) On the basis of the ecological chain design with reversed-planning theory, we are supposed to plan for nonconstructive land and restricted construction land by introducing concepts such as the connectivity of natural ecological units to ensure that the urban lake area can 
function well in ecological level;

3) To carry out the planning of constructive land based on the project of non-constructive land and restricted construction land;

4) In the end, to synthetically ameliorate the established planning and design planning for the sake of forming the ultimate plan.

\section{A design case study using Yunlong Lake}

Taking Yunlong Lake as an instance, the planning and design are carried out in accordance with the workflow that combines ecological chain and reversed-planning theory (Fig. 3). To start with, the research analyses and classifies the environmental ecological chain of Yunlong Lake and its surrounding areas, and then divide the area into four ecological zones of lake water system, lake peripheral wetland, peripheral mountain forest, and cities in the boundary of ecological chain. These four ecological zones take the lake water system as the core zone which contains four levels, including the bottom biological layer, the middle biological layer, the surface biological layer and the lakeshore biological layer. The ecological chain, composed of the four biological layers, sustains the natural ecology of the lake area and the layers in the ecological chain influence each other [9-10]. Diverse manual maintenance measures need to be taken before planning and designing the scenic area. For example, the bottom biological layer of the water system demands taking lake dredging measures, the middle and surface biological layers calls for the monitoring towards lake water quality, and the lakeshore biological layer needs to maintain a certain connectivity on environment of lake and shore to reduce the over-segmentation of artificial buildings in the natural environment. When planning a scenic spot, laying out with the goal of keeping great biodiversity can drastically level up the quality of ecological environment in scenic spot.

After the research and design of dividing functional zones of lake natural environment based on the ecological chain, according to the reversed-planning theory, nonconstructive land and restricted constructive land in scenic spots are obtained, and suitable constructive land is also gotten through reversing the background. In order to further consolidate the stable ecological pattern, each land and the connection space between them have been sorted out and transformed: biological corridors have been put into place, the division of artificial buildings on the lake shore to the natural environment has been decreased, and a considerable number of lake-land connections have been remained. In addition, by designing the island in the center of the lake, environmental functional segments such as the migratory bird conservation area and the amphibian breeding area have been added. In the area far away from city, the ecological zone of lake water system, the lake periphery wetland ecological zone and the peripheral mountain forest ecological zone are connected as one. The various ecological functions of urban lake scenic spots are all based on their original normal ecological environment. Hereby in designing, taking the natural environment as the first active element to design can better guarantee that the scenic spot achieves its diverse functions.

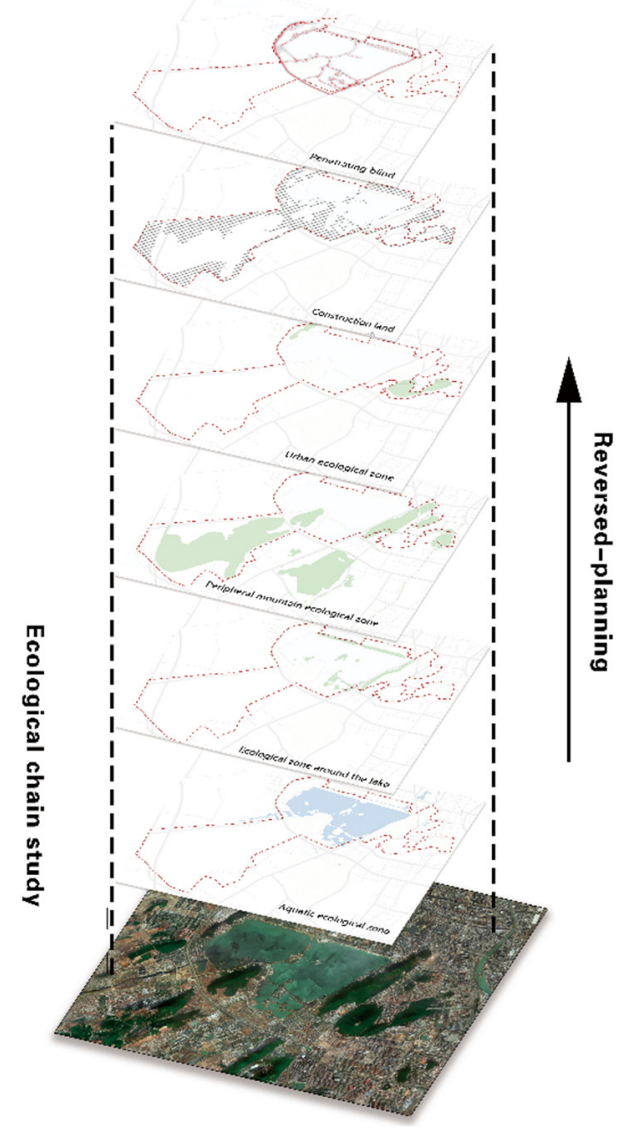

Fig. 3. Work flow of landscape design combining Reversedplanning and ecological chain

In the end, owing to perfecting the modification of Yunlong Lake's ecological function, the non-constructive land in the scenic land planning transforms to a lake ecological maintenance function zone, and the constructive land converts to a lake tourism and economic function zone. The latter can be further planned and divided into natural landscape sightseeing land and tourism expansion function land. In the specific design, the former mainly focuses on structures such as observation decks and submerged corridors, while the latter can be divided into cultural areas, catering area and retail area, etc. As for the next step of design, each structure should avoid cutting apart the natural environment, and the buildings ought to be concentrated as much as possible in their respective land for the sake of lessening the effect on the natural ecology of the lake. After obtaining the specific segmentations, the road network and the functional divisions are appropriately organized and regulated to form an integrated scenic spot planning and design. In addition, the introduction of artificial intelligence technology will improve the planning effect [12-13].

\section{Conclusions and Prospects}

Based on the design concept of ecological chain and reverse-planning, three functions of tourism service, economy and ecology can be unified under the condition 
of ensuring the excellent ecological function of urban lake landscape area, which is conducive to the improvement of urban natural environment and social environment. After all, it is of great significance to maintain the ecological stability of scenic spots in the city. By this way, this paper proposes a new design process that combines ecological chain and reverse-planning. That is, through design and planning measures such as multi-ecological function zoning, impeccable ecological chain design, as well as a series of design and planning measures of ecological planning first and architectural planning second, the optimized function of lake scenic spots aims to provide scientific basis and reference for landscape planning, tourism development and environment protection.

With the continuous expansion of urban space, the destruction of urban landscape environment is increasingly serious. Once the scenic area is built, its impact on the environment will begin to play a role, resulting in ecological loss which is hard to recover. Therefore, it is extremely crucial to fully incorporate environmental factors into the design concept. Comprehensively studying the ecological chain of scenic spots is the basis of environmental planning of scenic spots, which provides scientific basis for avoiding environmental impact caused by planning. The reversedplanning theory ensures that environmental factors are given priority by changing the workflow. The combination of ecological chain and reversed-planning theory ensures both theoretical advantages and practical feasibility of the planning. In the future, we plan to apply the new workflow in more cases of urban scenic spot planning to further promote the new workflow. The application of artificial intelligence technology in this process is also an exciting research direction. Collecting the information of the original topography, biology and ecological function of the scenic spot, intelligently analysing the ecological function and relationship of the scenic spot, and carrying out the fine design and planning, will be able to protect the sustainable development of the environment to the greatest extent. Intelligent analysis based on experience or algorithm can also dramatically reduce the human cost of planning and design. The results of intelligent analysis based on the same algorithm is instrumental in enhancing the comparability of planning schemes. Moreover, the application of artificial intelligence technology in this process is an stimulating research direction. In the future ecological chain design, we should refine the regional bionic community design, including animal habitat design and planting design, to enrich species diversity and build a stable cycle of the system. Above all, the results of intelligent analysis based on the same algorithm can be beneficial to intensify the comparability of planning schemes.

\section{Acknowledgement}

The research is sponsored by the Innovation Training Program for Artificial Intelligence Research Institute College Students, China University of Mining and Technology (No. CSA2020-02), the National innovation training program for College Students of China University of Mining and Technology (No. 201910290034Z) and Liu Baojun Science Foundation for Geoscience Young Scholars (No. DMSMX2019002). The author Difei Zhao also thanks to the China Scholarship Council (CSC) for providing the CSC scholarship for occupational training at the University of Queensland.

\section{References}

1. L Zhang, C Huang, D Zhao, H Du, Q Lu, H Yu, Y Yu, Y Chen, M Zhang. Fresen Environ Bull 29, 10: 9095 9102 (2020) .

2. E Mertens. Atmos Environ. 33, 24: 4115-4123(1999).

3. C Nilon, M Aronson, S Cilliers, D Cynnamon, L Frazee, M Goddard, K Neill, D Roberts, K Stander, E Stander, P Werner, M Winter, K Yocom.. Bioence. 67, 4: 332-342(2017).

4. X Zhang, B Li, H Xu, M Wells, B Tefsen, Qin B.. Water Research. 151, 3: 500-514(2019).

5. Yan, D., Xia, J. Hube Forestry ence and Technology. 153, 5: 12-17(2008).

6. P Macnaghten, R Owen. Nature. 479, 11: 293293(2011).

7. A Tamguicht. Economics Bulletin, 15, 9: 1-10(2007).

8. R King, C Richardson, U Romanowicz. Ecosystems. 7, 1: 75-97(2004).

9. E Halfon, N Schito, R Ulanowicz. Ecological Modelling. 86, 1: 0-36(1996).

10. A Neumann, D Kim, G Perhar, G Arhonditsis. $J$ Environ Manage. 188, 3: 308-321(2017).

11. Y Kongjian, L Dihua. Annual meeting of Chinese Association for Science and Technology. 26-37(2002)

12. S Laura, G Inma, O Eva, G Cesar. Int J Artif Intell T. 18, 11: 717-738(2009).

13. J Fink, A Kobsa. Artif Intell Rev. 18, 9: 33-74(2002) 